represented by a practically adequate approximation as

$$
\eta=\frac{\alpha a^{3}}{12 A}-\frac{a \eta_{0}}{\pi}
$$

The slit viscosimeter is an extremely delicate instru. ment, when used with small diameters. Expanded films, such as those of oleic, myristic or pentadecylic acid, have very low viscosities, and for their measurement the value of $a$ should be $0.1 \mathrm{~mm}$. or less. For $0.1 \mathrm{~mm}$. the correction term is only -0.00003 surface poises.

The substrate correction, $-a \eta_{0} / \pi$, is added to the earlier equation. While the highest viscosity $(0.0018$ surface poises for arachidic acid) given by Harkins and Myers is essentially correct, the lower values are too large by more than the experimental error. The correction applicable to their slit of negligible depth is smaller than the term given above, so to insure accurate results the work is being repeated by the use of a slit which meets the demands of the theory.

In the earlier letter, a ring viscosimeter, which vibrates as a torsion pendulum, was described. In addition, there is now in use a surface ring which rotates at a constant rate, either without or with a guard ring, the latter being used to simplify the theory. While theories may be developed for these instruments for the calculation of the absolute viscosity, the theories are somewhat doubtful, so it seems better to calibrate these instruments by the use of the slit viscosimeter.

William D. Harkins.

JoHN G. KIRKWOOD.

University of Chicago. Nov. 16.

${ }^{1}$ NATURe, 140, 465 (1937).

\section{Sorption of Carbon Dioxide by Chlorophyll}

THE interaction of chlorophyll with carbon dioxide is of obvious interest in connexion with the problem of photosynthesis. The formation of an addition product of chlorophyll and carbonic acid has been often assumed to be the first stage in the reduction process.

We performed some experiments to see if solid chlorophyll has any affinity for carbon dioxide. The preparation used was crystalline ethyl chlorophyllide ( $a+b$ mixture, $0 \cdot 3 \mathrm{gm}$. kindly supplied by Prof. A. Stoll of Basle). It was degassed in high vacuum at $70-80^{\circ} \mathrm{C}$. and brought into contact with pure carbon dioxide gas under different pressures. A considerable sorbing capacity was found. The sorption is perfectly reversible, and the equilibrium is established in a few seconds. The sorption isothermals for $0^{\circ} \mathrm{C}$. and $-80^{\circ} \mathrm{C}$. were measured out, point by point, in the interval $p=0$ to $700 \mathrm{~mm}$. They are perfectly reproducible smooth curves, and can be represented by the sorption formula derived previously ${ }^{1}$ for the sorption of gases by crystalline lattices ('zeolithic' sorption). The heat of sorption is $2 \cdot 6 \mathrm{kcal}$. per mol, and the saturation corresponds to 2 mols carbon dioxide per mol of ethyl chlorophyllide. The maximum sorption actually observed $\left(-80^{\circ} \mathrm{C}, p=600\right.$ $\mathrm{mm}$.), was $1.1 \mathrm{mols} / \mathrm{mol}$; at $0^{\circ}$ and $p=\frac{1}{4} \mathrm{~mm}$. (partial pressure of carbon dioxide in the air), about 1 ethyl chlorophyllide molecule in 15,000 is carrying a carbon dioxide molecule.
The relation of these results to the experiments of Willstätter and Stoll ${ }^{2}$ on sorption of carbon dioxide by colloidal chlorophyll solutions will be discussed elsewhere, together with the details of our experiments.

I wish to thank Mr. K. W. Jones for his help in carrying out the experiments.

Sir William Ramsay Laboratories of

Inorganic and Physical Chemistry, University College,

London.

Dec. 5.

${ }^{1}$ Rabinowitch, E., and Wood, W. C., Trans. Faraday Soc., 32, 947 (1936). In formulæ (4) and (6) of this paper, $R^{3 / 2}$ is a misprint for $R^{5 / 2}$. ${ }^{2}$ Willstätter, R., and Stoll, A., "Untersuchungen über die Assimilation der Kohlensaüre", pp. 227-314 (Berlin, 1918).

\section{Mechanism of the Biological Synthesis of Acetyl- choline}

THE well-known work of Prof. O. Loewi and of Sir Henry Dale and their collaborators has established the widespread function of acetylcholine as a chemical transmitter of nervous action. It has further been established that there exists in the animal organism a specific enzyme, choline-esterase, which effects the rapid destruction of this ester. Interest, therefore, now centres round the mechanism whereby acetylcholine is produced in the body.

Making the assumption that the synthesis in question occurs in nervous tissue, we recently ${ }^{1}$ succeeded in demonstrating conclusively that acetylcholine is produced in minced brain from the ox when this tissue is incubated under certain specified conditions. We further obtained some evidence which suggested that acetoacetic acid or one of its derivatives could act as a precursor of the acetyl moiety of the acetylcholine molecule, but the magnitude of the increase in the yield of acetylcholine produced by the addition of sodium acetoacetate to the incubating material was not sufficiently great to justify us in concluding with certainty that this substance acted as an immediate precursor.

Further progress has now been made in the elucidation of this problem. An examination of different portions of the brain by the technique previously described has shown that the synthesis of acetylcholine in vitro occurs to a preponderating extent, although not exclusively, in the grey matter, and that rather more is produced by the basal ganglia than by the grey cortical tissue. In view of this result and of the ease with which the basal ganglia can be removed from the remainder of the brain, we have now used this material in place of whole brain for ascertaining the influence of sodium acetoacetate on the synthesis of acetylcholine. The effect of the addition to this tissue has proved to be much greater than was previously observed with whole brain. In experiments which have been repeatedly carried out, the addition of $20 \mathrm{mgm}$. of sodium acetoacetate to $10 \mathrm{gm}$. of basal ganglia has increased the yield of acetylcholine, as determined by its action on the dorsal muscle of the leech, by 100 per cent. Further, although smaller, increases are produced by the addition of $10 \mathrm{mgm}$. of choline chloride.

These results appear to us to demonstrate that acetylcholine is synthesized biologically from choline and acetoacetic acid essentially according to the hypothetical scheme which we previously outlined, although they do not exclude the possibility that 